Instituto Internacional de Investigación y Desarrollo Tecnológico Educativo INDTEC, C.A.

DOI: https://doi.org/10.29394/Scientific.issn.2542-2987.2019.4.13.7.138-159

OAI-PMH: http://www.indteca.com/ojs/index.php/Revista Scientific/oai

Artículo Original / Original Article

\title{
El acento: Un estudio de creencias en estudiantes iniciales de Pedagogía en Lengua Inglesa
}

Autores: Luís Felipe Casimiro Perlaza Universidad Austral de Chile, UACh luis.casimiro@uach.cl

Valdivia, Chile https://orcid.org/0000-0001-7640-9038

Carolina Virginia Fuentes González Universidad Austral de Chile, UACh carolina.fuentes@uach.cl Valdivia, Chile https://orcid.org/0000-0001-8345-6481

\section{Resumen}

Desde hace décadas la enseñanza del idioma inglés como lengua extranjera ha estado polarizada haciendo que se crea que solo existen dos versiones de esta lengua: americana y británica. Tal polarización ha generado que los estudiantes no nativos del inglés, en general, crean que deben tener alguno de estos dos acentos como muestra de dominio del idioma. El presente estudio explora las creencias respecto al acento en un grupo de estudiantes iniciales de la carrera de Pedagogía en Lengua Inglesa de una universidad chilena. En el marco teórico se abordan las creencias del profesor en todos sus aspectos. La metodología es de tipo descriptiva con diseño de campo y nivel no experimental. Se realizó la adaptación del Instrumento Especializado para los estudios de Medición BALLI (Beliefs about language learning inventory) propuesto por Horwitz (1999), conformado por dos dimensiones: creencias basadas en la valoración y reacción frente a las variedades del idioma inglés haciendo énfasis en el acento. Los resultados obtenidos permiten concluir que, en el proceso de internacionalización del inglés, un usuario no nativo puede comunicarse perfectamente de forma "inteligible", respetando las normas del idioma, con el acento de su lengua nativa u otro, sin perjuicio de su identidad y origen.

Palabras clave: formación profesional; creencia; lengua.

Fecha de Recepción: 07-03-2019
Fecha de Aceptación: 19-06-2019
Fecha de Publicación: 05-08-2019 


\title{
The accent: A study of beliefs in initial students of Pedagogy in English Language
}

\begin{abstract}
For decades the teaching of English has a foreign language has been polarized making people believe that there are only two versions of this language: American and British. Such polarization has meant that non-native English students, in general, believe that they should have one of these two accents as a sign of language proficiency. The present study explores the beliefs regarding the accent in a group of initial students of English Language Pedagogy from a Chilean university. In the theoretical framework, the teachers' beliefs in all aspects are addressed. The methodology is of descriptive type with field design and non-experimental level. The adaptation of the Specialized Instrument for BALLI studies Measurement (Beliefs about language learning inventory) proposed by Horwitz (1999) was made, consisting of two dimensions: beliefs based on the assessment and reaction to varieties of the English language emphasizing the accent. The results obtained allow us to conclude that, in the process of internationalization of English, a non-native user can communicate perfectly in an "intelligible" way, respecting the rules of the language, with the accent of their native language or another, without prejudice to their identity and origin.
\end{abstract}

Keywords: vocational training; belief; language.

Date Received:

07-03-2019
Date Acceptance:

19-06-2019
Date Publication:

05-08-2019 


\section{Introducción}

El aprendizaje del inglés comprende el conocimiento y dominio de distintos aspectos propios de esta lengua, tales como: gramática, sintaxis, vocabulario, lenguaje corporal, cultura y pronunciación. En la actualidad muchos profesores y estudiantes de Inglés como Lengua Extranjera (ILE), son conscientes de las diferencias existentes en las formas del lenguaje de este idioma. Tales diferencias, en primera instancia, son percibidas por el acento que caracteriza a cada país, en modo general, si aprenden americano o británico. Desafortunadamente, en países hispanohablantes otros acentos en inglés han recibido poca o casi ninguna valoración al momento de aprender este idioma. En segunda instancia, otras de las diferencias que denotan tanto profesores como estudiantes son la gramática, el vocabulario y la cultura.

Los intercambios internacionales y la expansión de las tecnologías de información y comunicación que se han generado producto de la globalización han permitido que diferentes formas del lenguaje en inglés distintas a la británica o americana sean conocidas en el mundo de habla hispana. Sin embargo, durante los últimos treinta años la participación de Estados Unidos en el escenario internacional ha hecho que se produzca una diseminación de esta forma del lenguaje, lo que ha hecho que usuarios de otras lenguas tengan contacto con esta, encontrándola más fácil para comunicarse. No obstante, en el campo de la enseñanza del inglés predomina la suposición de que la variedad que debe ser enseñada es la británica, por considerarse la más correcta. A tal efecto, algunos profesores han recomendado un cambio de la variedad del inglés americano al británico, dejando a un lado las demás variedades de esta lengua.

Es conveniente resaltar que no existe una respuesta realmente verdadera respecto a qué variedad enseñar o qué acento aprender. Ciertamente el factor geográfico ha recibido gran importancia, por un lado, en México, por ejemplo, muchas personas tienen mayor posibilidad de estar en 
contacto con estadounidenses que con británicos, lo que cual es razonable. Por otro lado, en Italia, España o países asiáticos la posibilidad de contacto es mayor con británicos. En este orden de ideas, otro factor que entra en juego en el campo de la enseñanza es considerar si los estudiantes oirán o leerán materiales en la variedad americana, británica o alguna otra, caso que casi nunca ocurre.

En Chile, como país hispanohablante, se forman profesores de inglés como lengua extranjera. Sin embargo, distintas ofertas laborales destacan la necesidad de contratar profesores nativos americanos o británicos, dejando a un lado a los no nativos por no tener alguno de estos acentos. Ello es la expresión máxima de la valoración que se tiene de estas variedades del idioma y de quien enseña por su acento y no por el dominio de lengua y en especial de la didáctica. Esto constituye una muestra de un sistema de creencias que se convierte a la vez en una práctica de discriminación. Bajo este contexto, se promueve un conjunto de creencias en donde se juzga el desempeño del profesor de inglés por el acento que tenga, muchas veces, por encima de su desempeño lingüístico y profesional. Al respecto, Peña (2018): indica que no se debe pensar solo en el proceso de aprendizaje, sino en el aspecto del ser humano, como persona, basado en una educación integral y para la vida en función de los términos de la propia realidad de los estudiantes (pág. 218).

Desde una perspectiva sociolingüística, es necesario mencionar que el docente de inglés como lengua extranjera, como ser social, aprende y usa el idioma como instrumento para construir su relación con el mundo y la realidad que lo rodea más allá de sus fronteras. Por lo anterior, Chacón (2010a), destaca que, en la etapa inicial de formación docente, los estudiantes de pedagogía en lengua inglesa:

Construyen la identidad profesional con base en el sistema de creencias culturales que les comunican y constituyen la identidad colectiva profesional en sus diversas comunidades de práctica y que estas creencias dan forma a la identidad en 
cuanto a quiénes son, cómo se ven ellos mismos y cómo ven a los demás miembros de la comunidad donde participan y negocian significados (pág. 28).

Esta idea permite entender que la formación docente, y en particular, la constitución de la identidad profesional es un proceso continuo que va desde el inicio de la formación profesional y que sigue posterior al egreso universitario, a lo largo del desarrollo de la vida docente.

En relación al acento, Chacón (2010b): considera que es indivisible con la identidad que los profesores de inglés crean durante su formación; de la apreciación de sí mismos y de los demás, y de cómo creen ser percibidos o valorados con respecto a su competencia lingüística en el idioma (pág. 28). Esto puede verse reflejado en las palabras de Shin (2006), citado por Yi (2008), quién señala que: "el buen inglés es a menudo definido como la variedad de inglés hablado por los angloparlantes de países occidentales" (pág. 17). Para Lippi-Green (1997): el acento es una marca fonológica que permite identificar al individuo por su procedencia geográfica. Desafortunadamente, existe una tendencia a la práctica social de descalificar o desvalorar aquellos acentos del idioma inglés que no provienen de países conocidos como "grandes imperios" como: Inglaterra, Canadá, Australia, y Estados Unidos (pág. 42). Tales acciones constituyen una base ideológica que genera estereotipos y discriminación hacia los hablantes no nativos. Dicha idea toma fuerza en los países hispanohablantes donde se considera que el inglés británico es la versión "pura" o "elegante" de este idioma. Tal supuesto se ve reflejado en los profesores de inglés en formación no sólo acerca de sí mismos y de sus pares, sino también de los docentes que les imparten clases; hace que se desvalore o desestime otros acentos de esta lengua y genera que muchos estudiantes prefieran aprender el idioma con profesores cuyo acento sea el británico.

En resumen, la formación inicial de profesores de inglés como lengua extranjera debe estar destinada a ofrecer un conjunto de experiencias 
docentes que le permita a los estudiantes construir un sistema de creencias y su propia identidad como hablantes no nativos de esta lengua, con una visión valorativa "positiva y sólida" de quienes son, con sentido de pertenencia de su origen, con una visión multi e intercultural, con amplio reconocimiento como hablantes competentes de esta lengua extranjera y del rol que cumplirán una vez titulados. Todo esto, independiente del acento que escojan hablar.

De acuerdo a lo planteado, el presente estudio tiene como objetivo explorar las creencias respecto al acento en un grupo de estudiantes iniciales de la carrera de Pedagogía en Lengua Inglesa de una universidad chilena.

\section{Bases Teóricas}

\subsection{Creencias del Profesor}

La enseñanza es un proceso complejo que puede ser conceptualizado de diversas formas. Tradicionalmente, la enseñanza de idiomas ha sido descrita en términos de lo que el profesor hace, es decir, la acción y conducta que muestran en el aula y el efecto de ello en sus aprendices. De acuerdo a Richards (1994a): no importa qué tipo de clase enseñe el profesor, su desempeño sigue las siguientes tareas: Selección de actividades para el aprendizaje, preparación de los estudiantes para nuevos aprendizajes, presentación de actividades, realización de preguntas, conducción de ejercicios, chequeo de la comprensión de los estudiantes, monitoreo de los estudiantes, realización de retroalimentación a los estudiantes, realización de repaso y repetición cuando sea necesario (pág. 29).

Dado que se trata de entender cómo los profesores se relacionan con estas dimensiones de la enseñanza, es necesario que se examinen las "creencias" y procesos del pensamiento, los cuales subyacen en las acciones que el profesor lleva a cabo en el aula de clase. Al respecto, Clark y Peterson (1986); y Lynch (1989): indican que la enseñanza comprende una dimensión cognitiva, afectiva y conductual. Para Richards (1994b): esto se basa en la 
reflexión que hacen los profesores en cuanto a lo que saben y creen, y que el conocimiento y su pensamiento les proporcionan un esquema o base para la guía de sus acciones en el aula (pág. 29).

\subsection{La fuente de creencias del profesor}

Las creencias del profesor como sistema son fundadas en metas, valores y las creencias mismas acerca de la relación que tiene el contenido con los procesos de enseñanza y la comprensión de los sistemas con los que trabaja y su rol dentro del mismo. Estas creencias y valores sirven como antecedentes en la toma de decisión del profesor y su acción, pues constituye lo que se denomina "la cultura de la enseñanza".

Al respecto, Feiman-Nemser y Floden (1986): señalan que la cultura de la enseñanza está compuesta por las creencias relacionadas al trabajo y conocimientos que los profesores comparten, creencias acerca de las formas apropiadas de actuar en el trabajo y el apego a ciertos aspectos de la enseñanza, y el conocimiento que los motiva y les permite hacer su trabajo (pág. 508). Para Richards (1994c), el estudio del pensamiento del profesor está orientado a las siguientes preguntas: ¿Qué creen los profesores acerca de la enseñanza y el aprendizaje?, ¿Cómo organizan su conocimiento?, ¿Cuáles son las fuentes de sus creencias? y ¿Cómo sus creencias influencian su enseñanza? (pág. 30).

Las creencias del profesor son sistemas que básicamente se construyen a través del tiempo y que consisten en dimensiones las cuales pueden ser objetivas o subjetivas:

1. Su experiencia como estudiante: todo profesor alguna vez fue estudiante, y sus creencias acerca del aprendizaje son a menudo la expresión de cómo fueron enseñados.

2. Experiencia en lo que resulta mejor: para muchos profesores la 
experiencia es la fuente primaria de sus creencias acerca de la enseñanza. Un profesor puede darse cuenta de las estrategias de enseñanza que dan mejores resultados y las que no lo hacen.

3. Establecimiento de la práctica: dentro de una institución educativa ciertos estilos de enseñanza y prácticas son "deseados", y basado en ello, el profesor debe adaptar su actividad de clase.

4. Factores de la personalidad: algunos profesores demuestran una inclinación personal por un patrón particular de enseñanza, arreglo o actividad porque se relaciona con su personalidad.

5. Basamento teórico y principios: los profesores pueden orientarse a comprender los principios del aprendizaje desde una perspectiva de la psicología, adquisición de un segundo idioma o de la educación y tratar de aplicarla en el salón.

6. Principios derivados de un enfoque o método: algunos profesores creen en la efectividad de un enfoque o método de enseñanza y consistentemente intentan implementarlo en clase.

\subsection{Enseñanza de la pronunciación en inglés como lengua extranjera}

Uno de los objetivos que buscan alcanzar la mayoría de los profesores de inglés como lengua extranjera es que los estudiantes sean capaces de comunicarse de forma oral en esta lengua. No obstante, durante las décadas 60 y 70, los enfoques cognitivos propuestos para la enseñanza de lenguas extranjeras valoraban el aprendizaje de la gramática y el vocabulario por sobre la comunicación oral y la pronunciación, debido a que se creía que era imposible que los estudiantes alcanzaran un acento nativo.

En la actualidad, el enfoque de enseñanza mayormente utilizado para la enseñanza de inglés como lengua extranjera es el comunicativo. Al respecto, Levis (2005): señala que existen dos principios que han influenciado particularmente tanto la enseñanza de la pronunciación como la investigación 
en la misma; por un lado, el principio del acento nativo, que prioriza el acercamiento y, en lo posible, la apropiación de un acento nativo en la segunda lengua; y, por otro, el de la inteligibilidad, que pone el foco en la comunicación efectiva, más que en la adquisición de un acento. Dentro del enfoque comunicativo lo que prima es el mensaje, permitiendo la existencia de un acento no similar al de un hablante nativo, toda vez que no se vea obstaculizada la comunicación (pág. 370); y debido a que hoy por hoy, el número de hablantes de inglés como lengua extranjera es mayor al número de hablantes nativos, la comunicación puede darse con acentos de diferente índole, pues el enfoque que se sostiene es basado en la comunicación y no en la pronunciación, priorizando así el principio de la inteligibilidad.

Al respecto, Laroy (1995): indica que "el habla de los aprendices -su acento- es la expresión de su identidad y que los profesores deben respetarla" (pág. 7). En el caso de la formación de profesores de inglés como lengua extranjera, se debe conceptualizar una clara diferencia entre pronunciación y acento: por un lado, la pronunciación se caracteriza por la realización oral del lenguaje, sin menoscabo o perturbación del significado; por el otro, desde una perspectiva sociolingüística, el acento es la realización oral del lenguaje por parte de un individuo, región o país de forma peculiar.

Tanto los hablantes nativos tanto de inglés como de español, u otras lenguas, tienen un acento. Este es un aspecto que refleja las diferencias tanto lingüísticas como culturales de estas comunidades de habla y que está estrechamente ligado a la identidad y sentido de pertenencia. En este sentido, en Chile, la formación de profesores está orientada a capacitar profesionalmente a docentes de habla hispana para que ejecuten actividades didácticas en inglés como lengua extranjera con alto nivel de desempeño lingüístico y profesional. Sin embargo, prevalece la creencia de que el profesor no nativo de inglés no debería mostrar marcas de acentos locales al momento de hablar en inglés. Esto podría ser producto de las demandas sociales en 
esta materia, las cuales son supuestos que se creen como verdad al pensar que con un profesor nativo de la lengua inglesa realmente se puede aprender hablar este idioma.

Por lo anterior, es necesario que se fortalezca la identidad personal y profesional del profesor de inglés en formación para que éste se auto reconozca como mediador intercultural y lingüístico independiente del acento que tenga al momento de hablar en el idioma extranjero. Ya diversas teorías y enfoques han dado cuenta que no todos los estudiantes de idiomas extranjeros logran adquirir un acento similar al nativo, pero si un "dominio maestro" de la lengua como se establece el Marco Común Europeo de Referencia para las Lenguas propuesto por el Consejo de Europa (2001): "el dominio maestro de una lengua extranjera es caracterizado por el grado de precisión, propiedad y facilidad en el uso de la lengua que tipifica el habla de los alumnos brillantes" (pág. 39). En este sentido, los estudiantes deben ser informados desde el comienzo que lo que realmente importa es que pronuncien bien en inglés, independientemente si su acento suena americano, británico o extranjero, porque la mayor relevancia subyace en la comunicación con una pronunciación inteligible; es decir, que los mensajes sean expresados de forma comprensible. Pues, si los estudiantes son informados de ello, sus niveles de estrés disminuirán notablemente, ya que siempre serán no nativos en la lengua extranjera y nativos en la suya. La adquisición de un acento en inglés como lengua extranjera es un proceso complejo y sobre todo personal de los estudiantes, quienes tienen el derecho de elegir entre las diferentes variedades: americano, británico, australiano, trinitario, entre otros, o extranjero conservando el acento de su lengua materna. Esta última opción está en consonancia con el proceso de internacionalización del idioma inglés y de la cultura e identidad de los hablantes nativos de otras lenguas que son reflejadas por marcas de su acento nativo y que no deben ser discriminados 0 juzgados negativamente por ello. 


\section{Metodología}

La metodología empleada para llevar a cabo el presente estudio es de tipo descriptiva, pues explora las creencias respecto al acento en un grupo de estudiantes iniciales de Pedagogía en Lengua Inglesa de una universidad chilena. Para Bernal (2016a): la investigación de tipo descriptivo es aquella en donde "se muestran, narran, reseñan o identifican hechos, situaciones, rasgos, características de un objeto de estudio" (pág. 143).

Igualmente, la investigación se caracteriza por tener un diseño de campo y transeccional descriptivo. Para Bernal (2016b): "la información del objeto de estudio se obtiene en una única vez en un momento dado" (pág. 145). Se considera de campo porque los datos fueron recolectados en el marco del contexto una institución de educación superior universitaria chilena. Por tratarse de la exploración de las creencias sostenidas por el grupo de estudiantes en cuestión, la investigación es no experimental; ya que no se pretende alterar sus creencias.

Se realizó la adaptación del Instrumento Especializado para la Medición Beliefs about language learning inventory, propuesto en su revisión de estudios BALLI por Horwitz (1999): el cual permite entender o comprender las creencias de los estudiantes respecto al aprendizaje de una lengua extranjera (págs. 557-576).

La adaptación de dicho instrumento está conformada por un total de dos dimensiones: creencias basadas en la valoración y reacción frente a las variedades del idioma inglés haciendo énfasis en el acento. Para ello se otorgaron veinte proposiciones para obtener la valoración y la descripción de seis acentos preseleccionados a fin de conocer las reacciones de los encuestados. En cada proposición los estudiantes debían dar su valoración tomando en cuenta las siguientes opciones: Totalmente de acuerdo (TA), de acuerdo $(A)$, neutral $(N)$, en desacuerdo (D) y totalmente en desacuerdo (TD). Las reacciones de los encuestados respecto a los seis tipos acentos 
preseleccionados del idioma inglés se obtuvieron empleando adjetivos con significados tanto positivos como negativos. Las respuestas fueron tabuladas en términos positivos y negativos, según la frecuencia de selección.

Con relación a los índices de confiabilidad del instrumento Especializado para la Medición BALLI, el coeficiente obtenido para dicho inventario de creencias fue de 0,865 realizado a través del test de confiabilidad Alfa de Cronbach. El instrumento de recolección de la información fue administrado a un total de 50 estudiantes del primer semestre de la carrera de Pedagogía en Comunicación en Lengua Inglesa de una universidad chilena.

\section{Análisis de Resultados}

La presentación de los resultados obtenidos se hace en dos secciones: Valoración y descripción del acento en el idioma inglés. En cada una de estas secciones presentan tablas con su respectiva explicación de los resultados.

\subsection{Valoración del acento en el idioma inglés}

En la tabla 1, se presenta el primer ítem del instrumento requirió que los encuestados expresaran su valoración a 20 proposiciones referidas al acento en el idioma inglés.

Tabla 1. Valoración del acento en el idioma inglés.

\begin{tabular}{|l|c|c|c|c|c|c|}
\cline { 2 - 7 } \multicolumn{1}{l|}{} & TA & A & N & D & TD & TOTAL \\
\hline El acento británico es mejor que el americano. & 1 & 5 & 27 & 9 & 8 & 50 \\
\hline El acento americano es mejor que el británico. & 1 & 2 & 27 & 13 & 7 & 50 \\
\hline $\begin{array}{l}\text { El acento de las islas del caribe es peor que el } \\
\text { americano y el británico. }\end{array}$ & 0 & 1 & 24 & 16 & 9 & 50 \\
\hline El acento americano es informal. & 0 & 15 & 12 & 12 & 11 & 50 \\
\hline El acento británico es el original. & 3 & 15 & 22 & 7 & 3 & 50 \\
\hline El acento irlandés es difícil de entender. & 7 & 28 & 10 & 3 & 2 & 50 \\
\hline El acento hindú es difícil de entender. & 6 & 23 & 13 & 8 & 0 & 50 \\
\hline $\begin{array}{l}\text { El acento inglés de hablantes asiáticos es difícil de } \\
\text { entender. }\end{array}$ & 3 & 11 & 20 & 16 & 0 & 50 \\
\hline El acento británico es el que se debe enseñar en los & 1 & 4 & 26 & 15 & 4 & 50 \\
\hline
\end{tabular}




\begin{tabular}{|l|c|c|c|c|c|c|}
\hline cursos de inglés. & & & & & & \\
\hline $\begin{array}{l}\text { El acento americano es el que se debe enseñar en los } \\
\text { cursos de inglés. }\end{array}$ & 2 & 10 & 28 & 9 & 1 & 50 \\
\hline $\begin{array}{l}\text { El idioma inglés puede ser enseñado con un acento } \\
\text { diferente al americano o británico. }\end{array}$ & 10 & 22 & 7 & 8 & 3 & 50 \\
\hline $\begin{array}{l}\text { Para hablar correctamente en inglés se debe imitar un } \\
\text { acento nativo. }\end{array}$ & 2 & 3 & 12 & 16 & 17 & 50 \\
\hline $\begin{array}{l}\text { Es difícil hablar inglés con un acento que no sea } \\
\text { británico o americano. }\end{array}$ & 3 & 14 & 12 & 14 & 7 & 50 \\
\hline Los chilenos no tienen un buen acento en inglés. & 2 & 10 & 14 & 15 & 9 & 50 \\
\hline El acento en inglés de los latinoamericanos es malo. & 2 & 8 & 8 & 18 & 14 & 50 \\
\hline $\begin{array}{l}\text { Con un profesor no nativo del inglés no se puede } \\
\text { aprender un acento. }\end{array}$ & 0 & 2 & 4 & 12 & 32 & 50 \\
\hline $\begin{array}{l}\text { Un/a profesor/a nativo/a del español tiene que hablar } \\
\text { en inglés sin acento hispanoamericano. }\end{array}$ & 1 & 4 & 11 & 16 & 18 & 50 \\
\hline Me gusta mi acento en inglés. & 8 & 21 & 14 & 6 & 1 & 50 \\
\hline $\begin{array}{l}\text { Para adquirir un acento debo irme a un país de habla } \\
\text { inglesa. }\end{array}$ & 2 & 13 & 14 & 17 & 4 & 50 \\
\hline $\begin{array}{l}\text { Hablar en inglés con el acento de mi lengua materna } \\
\text { es incorrecto. }\end{array}$ & 2 & 6 & 6 & 21 & 15 & 50 \\
\hline
\end{tabular}

Fuente: Los Autores (2019).

Los resultados presentados en la Tabla 1, indican que los encuestados tienen una postura neutral respecto a considerar que el acento británico sea mejor que el americano o viceversa. Esta misma postura neutral se mantiene en relación a la valoración del inglés hablado en las islas del caribe. En lo que respecta a la informalidad del acento americano se observa que gran parte muestra una postura favorable al no considerarlo de tipo "informal". En cuanto a la originalidad del acento se observa que gran parte mantiene una postura neutral respecto a considerar el británico como "original". En relación a la dificultad de comprensión de los acentos irlandés e hindú, al menos, la mitad de los encuestados mostró estar de acuerdo en que estos dos acentos son difíciles de entender. Sin embargo, la mayoría mantiene una postura neutral en relación a la dificultad de comprensión del inglés hablado por asiáticos.

En lo que respecta a la selección de un acento en la enseñanza del idioma inglés, la mayoría mostró una postura neutral de valoración del acento americano y británico. Igualmente, se pudo observar que la mayoría está de 
acuerdo que el idioma inglés puede ser enseñado con acentos distintos al americano o británico. La mayoría no estuvo de acuerdo en que hablar correctamente en inglés requiera la imitación del acento de un hablante nativo. Igualmente, se observa que la mayoría considera que no es difícil hablar en inglés con un acento distinto al británico o americano. En cuanto a la calidad del acento en inglés de los chilenos y latinoamericanos la mayoría mostró estar en desacuerdo que este sea malo.

Similarmente, la mayoría mostró estar de acuerdo que se puede aprender el idioma con un profesor que no sea nativo y que no tenga acento nativo. Asimismo, se observa que la mayoría está conforme con su acento en inglés, y que además consideran que no es necesario viajar a un país de habla inglesa para mejorar el acento y que no es incorrecto hablar en inglés con trazos del acento nativo (hispano).

\subsubsection{Descripción al acento en el idioma inglés.}

El segundo ítem del instrumento recolectó las descripciones elaboradas por los encuestados respecto a seis acentos en inglés: americano, británico, caribeño, asiático, irlandés e hispano. Esto permitió medir las reacciones frente a cada uno de estos acentos, ya que algunas de las descripciones incluyeron palabras emocionales, peyorativas y fuertes.

\subsubsection{Inglés con acento americano}

En la Tabla 2, se observa que los encuestados mostraron reacciones favorables respecto al inglés con acento americano. Los encuestados cuyo gusto está a favor del acento británico se abstuvieron de hacer comentarios negativos respecto a este acento. 
Tabla 2. Descripción del inglés con acento americano.

\begin{tabular}{|l|l|}
\hline \multicolumn{1}{|c|}{ Reacciones Positivas } & Reacciones Negativas \\
\hline Comprensible & \\
Estándar & \\
Fluido & \\
Aceptable & \\
Claro & \\
Rápido & \\
Familiar & \\
\hline
\end{tabular}

Fuente: Los autores (2019).

\subsubsection{Inglés con acento británico.}

En la Tabla 3, se evidencia que algunos de los encuestados a quienes les gusta el inglés americano mostraron reacciones peyorativas respecto al inglés británico, por ejemplo: exagerado, difícil o confuso. Sin embargo, la mayoría mostró reacciones positivas frente a este acento.

Tabla 3. Descripción del inglés con acento británico.

\begin{tabular}{|l|l|}
\hline \multicolumn{1}{|c|}{ Reacciones Positivas } & \multicolumn{1}{c|}{ Reacciones Negativas } \\
\hline Comprensible & Exagerado \\
Muy bueno & Difícil \\
Bonito & Confuso \\
Melodioso & \\
De la clase social alta & \\
Agradable & \\
Educado & \\
Prestigioso & \\
\hline
\end{tabular}

Fuente: Los autores (2019).

\subsubsection{Inglés con acento caribeño}

En la Tabla 4, se percibe que las reacciones frente a este acento fueron relativamente balanceadas. Sin embargo, los encuestados cuyo gusto estuvo a favor del inglés americano o británico consideraron este acento como rápido, exagerado o escandaloso. 
Tabla 4. Descripción del inglés con acento caribeño.

\begin{tabular}{|l|l|}
\hline \multicolumn{1}{|c|}{ Reacciones Positivas } & \multicolumn{1}{c|}{ Reacciones Negativas } \\
\hline Comprensible & Rápido \\
Sexy & Escandaloso \\
Expresivo & Exagerado \\
Rítmico & \\
\hline
\end{tabular}

Fuente: Los autores (2019).

\subsubsection{Inglés con acento asiático}

En la Tabla 5, desafortunadamente se evidencia que la mayoría de los encuestados mostró reacciones negativas frente a este acento. Del mismo modo, pocos lo describieron como "comprensible".

Tabla 5. Descripción del inglés con acento asiático.

\begin{tabular}{|l|l|}
\hline \multicolumn{1}{|c|}{ Reacciones Positivas } & \multicolumn{1}{|c|}{ Reacciones Negativas } \\
\hline Comprensible & Incompresible \\
& Desconocido \\
& Confuso \\
& Retorcido \\
& Extraño \\
& Poco claro \\
& Raro \\
& Poco Familiar \\
\hline
\end{tabular}

Fuente: Los Autores (2019).

\subsubsection{Inglés con acento irlandés}

En la Tabla 6, se muestran las reacciones obtenidas por los encuestados frente a este acento, las cuales resultaron ser más severas en comparación con los demás. Pocos describieron el inglés irlandés como "comprensible". 
Tabla 6. Descripción del inglés con acento irlandés.

\begin{tabular}{|l|l|}
\hline \multicolumn{1}{|c|}{ Reacciones Positivas } & \multicolumn{1}{c|}{ Reacciones Negativas } \\
\hline Comprensible & Duro \\
& Rápido \\
& Desagradable \\
& Exagerado \\
& Poco claro \\
& Incomprensible \\
& Difícil \\
& Cansón \\
& Confuso \\
& Poco familiar \\
\hline
\end{tabular}

Fuente: Los autores (2019).

\subsubsection{Inglés con acento hispano.}

En la Tabla 7, dado que la lengua materna de los encuestados es el español, se pudo evidenciar reacciones más favorables respecto al inglés hablado con este acento. Sin embargo, algunos de los encuestados mostraron reacciones negativas como: lento, extraño, poco claro, de la clase baja y malo.

Tabla 7. Descripción del inglés con acento hispano.

\begin{tabular}{|l|l|}
\hline \multicolumn{1}{|c|}{ Reacciones Positivas } & \multicolumn{1}{c|}{ Reacciones Negativas } \\
\hline Comprensible & Lento \\
Muy bueno & Extraño \\
Claro & Poco claro \\
Bonito & De la clase baja \\
Fluido & Malo \\
Aceptable & \\
Familiar & \\
\hline
\end{tabular}

Fuente: Los autores (2019).

\section{Conclusiones}

Las creencias constituyen la expresión máxima de las experiencias de vida de cada individuo, las cuales le permitirán configurar su propia realidad haciendo que esta sea considerada como única, válida y verdadera. Sin 
embargo, dicha configuración de la realidad puede estar compuesta de supuestos e ideologías que no necesariamente sean ciertas. La globalización del idioma inglés ha estado polarizada desde hace décadas, pues en la mayoría de los casos solo se toman como referentes dos versiones de esta lengua: americana y británica. Esto deja poco espacio en las aulas de clase para que los estudiantes sean expuestos a otros acentos.

Por ello, es necesario que estos sean expuestos a diferentes versiones de la lengua inglesa, desde el inicio de sus estudios. Estas acciones permitirán la formación global de los mismos en el idioma, además que disminuiría notablemente los prejuicios respecto a aquellos acentos poco conocidos 0 comunes a escala mundial. Dado que el acento es una forma particular de pronunciar un idioma y que está estrechamente relacionada con la identidad de quienes lo hablan, se hace imperativo los estudiantes no nativos del idioma inglés tengan el poder de decidir bajo qué acento hablarán en esta lengua.

En otras palabras, la adquisición de un acento no puede ser un proceso forzado ni confundido con un indicador de dominio competente de la lengua. Además, debido al proceso de internacionalización del inglés, un usuario no nativo puede comunicarse perfectamente de forma "inteligible", respetando las normas del idioma, con el acento de su lengua nativa u otro, sin perjuicio de su identidad y origen.

\section{Referencias}

Bernal, C. (2016a,b). Metodología de la investigación: Administración, Economía, Humanidades y Ciencias Sociales. 4ª edición, ISBN: 978958-699-309-8. Bogotá, Colombia: Pearson Educación, 384 págs.

Chacón, C. (2010a,b). Acento e identidad profesional en la formación del docente de inglés: Una perspectiva crítica. Revista Paradigma, 31(2), 25-36, e-ISSN: 1011-2251. Recuperado de: 
Clark, C., \& Peterson, P. (1986). Teachers' Thought Processes. In: Wittrock, M.C., Ed., Handbook of Research on Teaching, 3rd Edition, New York, EE. UU.: Macmillan Education, pp 255-296.

Consejo de Europa (2001). Marco común europeo de referencia para las lenguas: aprendizaje, enseñanza, evaluación. Primera Edición 2002, ISBN: 84-667-1618-1. Madrid, España: Secretaría General Técnica del MECD-Subdirección General de Información y Publicaciones, y Grupo ANAYA, S.A. Recuperado de:

https://cvc.cervantes.es/ensenanza/biblioteca ele/marco/cvc mer.pdf

Feiman-Nemser, S., \& Floden, R. E. (1986). The cultures of teaching. In M. C. Whittrock (Ed.), Handbook of research on teaching (3rd ed., pp. 505526). London, England: Collier-Macmillan.

Horwitz, E. (1999). Cultural and situational influences on foreign language learners' beliefs about language learning: a review of BALLI studies. System, 27(4), 557-576, e-ISSN: 0346-251X. Recuperado de: https://doi.org/10.1016/S0346-251X(99)00050-0

Laroy, C. (1995). Pronunciation. ISBN: 0-19-437087-9. New York, EE. UU.: Oxford University Press.

Levis, J. (2005). Changing contexts and shifting paradigms in pronunciation teaching. Tesol Quarterly, 39(3), 369-377, e-ISSN: 1545-7249. Recuperado de: https://doi.org/10.2307/3588485

Lynch, T. (1989). Researching teachers: behavior and belief. In C. Brumfit and R. Mitchel (eds): Research in the Language Classroom. London, England: Modern English Publications and the British Council.

Lippi-Green, R. (1997). English with an Accent: Language, Ideology and Discrimination in the United States. 2nd Edition, ISBN 10: 0-41555911-1; ISBN 13: 978-0-415-55911-9. New York, EE. UU.: Routledge. Peña, J. (2018). Transformación del Docente desde el Pensamiento Complejo. Revista Scientific, 3(7), 211-230, e-ISSN: 2542-2987. 
Recuperado de:

https://doi.org/10.29394/Scientific.issn.2542-2987.2018.3.7.11.211-230 Richards, J. (1994a,b,c). Reflective Teaching in Second Language Classroom. First Edition, ISBN: 978-0-521-45181-9. USA, EE. UU.: Cambridge Language Education.

Yi, Á. (2008). Cambios de paradigma en la enseñanza de inglés como lengua extranjera el cambio crítico y más allá. Revista Educación y Pedagogía, 20(51), 11-23, e-ISSN: 0121-7593. Recuperado de:

https://dialnet.unirioja.es/servlet/articulo?codigo $=3074193$ 


\section{Luís Felipe Casimiro Perlaza}

e-mail: luis.casimiro@uach.cl

Nacido en Caracas, Venezuela, el 13 de febrero del año

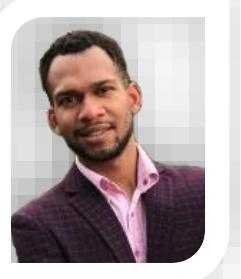
1988. Doctor Interdisciplinario en Ciencias: especializado en Neurodidáctica, Lingüística Aplicada y Ciencias de la Educación de la Universidad Simón Bolívar (USB) de Venezuela; Especialista en Enseñanza de Idiomas Extranjeros: Inglés de la Universidad Simón Bolívar (USB); Experto en Lenguaje y sus aplicaciones de la Universidad Simón Bolívar (USB); Licenciado en Administración; Licenciado en Educación mención: Docencia en Idioma Inglés. Me desempeño como Profesor de Planta Académica en el Programa de Pedagogía en Comunicación en Lengua Inglesa, adscrito al Instituto de Lingüística y Literatura de la Universidad Austral de Chile (UACh). 


\section{Carolina Virginia Fuentes González}

e-mail: carolina.fuentes@uach.cl

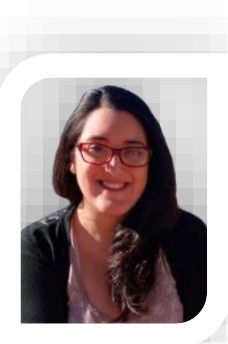

Nacida en Valdivia, Chile, el 17 de junio del año 1992.

Profesora en Comunicación en Lengua Inglesa, con mención en Traducción y en Técnicas Teatrales para la Enseñanza del Inglés como Lengua Extranjera de la Universidad Austral de Chile (UACh); Licenciada en Educación y Bachiller en Humanidades y Ciencias Sociales. Me desempeño como Profesora de inglés como lengua extranjera en Educación Prescolar y como Profesora de Planta Docente en el Programa de Pedagogía en Comunicación en Lengua Inglesa, adscrita al Instituto de Lingüística y Literatura de la Universidad Austral de Chile (UACh). 